\title{
La comunicación de la innovación realizada por empresas españolas en 2016 y 2017 a través de las redes sociales digitales
}

\author{
Maria J. Vilaplana-Aparicio \\ Universidad de Alicante \\ maria.vilaplana@ua.es \\ https://orcid.org/0000-0002-6373-8454
}

\author{
Mar IgLesias-García \\ Universidad de Alicante \\ mar.iglesias@ua.es \\ https://orcid.org/0000-0001-7926-5746
}

\author{
Marta Martín-Llaguno \\ Universidad de Alicante \\ marta.martin@ua.es
}

https://orcid.org/0000-0003-2252-5505

\section{The communication of innovation carried out by spanish companies in 2016 and 2017 through social networks}

\section{RESUMEN ABSTRACT}

Esta investigación se centra en la comunicación de la innovación que las empresas realizan a través de las redes sociales digitales (RSD) más importantes en España. Mediante el análisis de contenido se estudia si las compañías que han recibido ayudas públicas de I+D+i utilizan las redes sociales para informar del apoyo logrado. Se observan los perfiles de las organizaciones en siete plataformas durante un marco temporal de dos años. Del estudio se desprende que la presencia de las empresas innovadoras en las redes sociales es modesta y que las RSD apenas se utilizan para complementar los requisitos de información y comunicación, contemplados en los textos legales de referencia. Teniendo

en cuenta el nivel de penetración de las redes sociales en

España es superior a la media mundial y que se trata de un medio adecuado para compartir información de interés público, sería de interés incluirlas dentro de las obligaciones de comunicación. Asimismo, su inclusión requeriría reflexionar sobre la información que se debe publicar, cuándo debe hacerse y qué plataformas emplea.

PALABRAS CLAVE

Redes Sociales Digitales; Difusión; Comunicación empresarial; Innovación; I+D+i
This research focuses on the communication of innovation that companies carry out through the most important digital social networks in Spain. Through content analysis, we study whether companies that have received public R\&D\&I aid use social media to report the support achieved. The profiles of organizations are observed on seven platforms during a two-year time frame. The study shows that the presence of innovative companies in social networks is modest and that them are hardly used to complement the information and communication requirements, contemplated in the legal reference texts. Taking into account the level of penetration of digital social networks in Spain is higher than the world average and that it is an adequate means to share information of public interest, it would be of interest to include them within the communication obligations. Also, its inclusion would require reflection on the information to be published, when it should be done and what platforms to use.

KEYWORDS

Digital social networks; Diffusion; Business communication; Innovation; RED 


\section{Introducción}

\subsection{La comunicación de la innovación solicitada por CDTI y la transparencia}

Las empresas españolas pueden acceder a diferentes ayudas públicas para sus proyectos de investigación, desarrollo e innovación tecnológica $(1+D+i)$. Una parte muy importante de estas ayudas las gestiona el Centro para el Desarrollo Tecnológico Industrial (CDTI), una Entidad Pública Empresarial, dependiente del Ministerio de Ciencia, Innovación y Universidades.

Todas las empresas que reciben ayudas de CDTI deben cumplir con una serie de obligaciones, entre las que se encuentra informar al público del apoyo obtenido (de CDTI y de los fondos europeos denominados Feder) e incorporar una breve descripción en el sitio de Internet, que sea proporcional al apoyo recibido y que incluya información sobre los objetivos y resultados del proyecto (Vilaplana-Aparicio, Martín-Llaguno e Iglesias-García, 2018). La difusión a través de las Redes Sociales Digitales no tiene carácter preceptivo y son las empresas las que deciden si quieren utilizarlas para incrementar el impacto de la comunicación.

Estos requisitos de comunicación no son un caso aislado. Todas las empresas que reciben una ayuda de $1+D+i$ deben realizar unas determinadas acciones que contribuyan a que el público tenga conocimiento de los proyectos. Así viene establecido en la legislación que regula las ayudas en España (Ley 38/2003; Real Decreto 887/2006; Ley 19/2013) y en Europa (Comunicación de la Comisión 2014/C 198/01; Reglamento No 1303/2013; Reglamento no 821/2014) para aumentar el nivel de transparencia en la asignación de fondos y el impacto de los proyectos.

La transparencia es un elemento fundamental para los estados de la Unión Europea. En España se ha elaborado un marco legal que regula la transparencia y el acceso a la información (Ley 19/2013) y se ha avanzado con la creación del Portal de la Transparencia de España y de la Base de Datos Nacional de Subvenciones, que contienen información relativa a las subvenciones convocadas y concedidas.

Sin embargo, son varios los expertos que puntualizan que hay deficiencias en el nivel de cumplimiento de los requisitos de transparencia. Por su parte, Cruz-Rubio (2017), tras estudiar el cumplimento de la Ley de Transparencia, concluye que las grandes empresas están publicando una información incompleta, dispersa y que difiere de una empresa a otra. Esto coincide con las contribuciones de Beltrán-Orenes y Martinez-Pastor (2017), que examinan la información del Portal de la Transparencia de España y concluyen que las entidades de carácter privado no cumplen con los principios marcados por la Ley de Transparencia.

\subsection{Las Redes Sociales Digitales en el mundo y en España}

Las Tecnologías de la Información y la Comunicación juegan un papel clave en la sociedad. El acceso a Internet ha marcado un antes y un después y las empresas son conscientes de que deben tener presencia (Ruiz, 2012).

Los datos lo avalan: en 2016 eran 3.419 mil millones de personas las que tenían acceso a internet en todo el mundo y esta cifra se ha incrementado hasta alcanzar los 4.388 mil millones de personas (We Are Social, 2016; We Are Social, 2019), lo que supone que más de la mitad de la población mundial ahora está en línea (tabla 1).

Las personas tienen un mayor acceso a internet y eso se traduce también en un mayor uso de las redes sociales. En 2016 el número de usuarios de las RSD era de 2.307 millones de personas y esta cifra ha crecido hasta alcanzar los $3.484 \mathrm{mil}$ millones en 2019, lo que supone que las redes sociales han pasado de tener un nivel de penetración del 31\% a tener el 45\% para el mismo periodo (tabla 1).

A nivel mundial Facebook es la plataforma que más usuarios tiene, alcanzando los 2.271 millones de usuarios en 2019, frente a los 1.590 millones de usuarios que tenía en 2016. Además, se sitúa por delante de otras plataformas como YouTube, Instagram, Twitter o Linkedln (We Are Social, 2016 y 2019).

En España, según los últimos datos registrados en enero de 2019, también hay un incremento significativo, pues se ha pasado de tener 22 millones de usuarios en 2016 a tener 28 millones en 2019. Es decir, durante los últimos cuatro años se ha incrementado el porcentaje de penetración de las redes sociales hasta lograr el 60\% (tabla 2).

A diferencia de lo que ocurre a nivel mundial, las redes sociales con más usuarios españoles desde 2017 son YouTube y

\begin{tabular}{|r|c|c|c|c|}
\hline & $\mathbf{2 0 1 6}$ & $\mathbf{2 0 1 7}$ & $\mathbf{2 0 1 8}$ & $\mathbf{2 0 1 9}$ \\
\hline Usuarios de Internet & 3.419 billones & 3.773 billones & 4.021 billones & 4.388 billones \\
\hline Usuarios RSD & 2.307 billones & 2.789 billones & 3.196 billones & 3.484 billones \\
\hline \% Penetración de las RSD & $31 \%$ & $37 \%$ & $42 \%$ & $45 \%$ \\
\hline
\end{tabular}

Tabla 1. Usuarios de las redes sociales digitales durante los últimos cuatro años. Elaboración propia a partir de We Are Social $(2016 ; 2017 ; 2018 ; 2019)$. 


\begin{tabular}{|r|c|c|c|c|}
\hline & 2016 & 2017 & 2018 & 2019 \\
\hline Usuarios RSD & 22 millones & 25 millones & 27 millones & 28 millones \\
\hline \% Penetración de las RSD en España & $48 \%$ & $54 \%$ & $58 \%$ & $60 \%$ \\
\hline
\end{tabular}

Tabla 2. Usuarios de las redes sociales en España durante los últimos cuatro años. Elaboración propia a partir de We Are Social $(2016 ; 2017 ; 2018 ; 2019)$.

Facebook (tabla 3), seguidas de otras redes sociales como, Instagram, Twitter, Google +, Linkedln y Pinterest (We Are Social, 2016, 2017, 2018 y 2019).

En definitiva, no cabe duda de que "las redes sociales se mantienen con buena salud" en España (IAB, 2017, p. 10) y que son un medio a tener en cuenta.

\subsection{Las RSD en el mundo empresarial}

Las Redes Sociales Digitales también están cambiando la forma en que se comunican las empresas, que tienen la posibilidad de personalizar los mensajes y de interactuar con sus públicos. Las empresas, conscientes del crecimiento de la actividad en las RSD, las están incorporando a su estrategia de marketing debido a su bajo coste y su popularidad. Sin embargo, actualmente el uso de estas redes por parte de las compañías todavía "responde más a una presencia testimonial que a un verdadero espacio de participación con las comunidades de usuarios", pues en general la frecuencia de actualización e interacción es baja (Sixto, Aguado y Riveiro, 2017, p. 65).

Según un estudio de la Fundación Telefónica (2014) centrado en las microempresas, Facebook es la red social más conocida y utilizada en el entorno empresarial, seguida por Twitter y Linkedln, las otras dos plataformas más empleadas. No obstante, no se realizan los mismos usos de todas las redes. Aunque Facebook es la plataforma más conocida, Twitter se usa en mayor proporción para fines profesionales y Linkedln es la red social en la que más empresas están presentes con una cuenta únicamente profesional. Además, predominan las entidades que tienen presencia en más de una red social (57\%).

Es tal el auge que están teniendo las RSD en la empresa, que especialistas como Andreu (2015) consideran que van a cambiar la forma en que las empresas se relacionan con sus públicos por los siguientes motivos:

- El stakeholder engagement o la forma en que las empresas dialogan con sus grupos de interés. Ahora tienen la posibilidad de interactuar con un grupo superior de personas y dar a conocer sus productos a nivel mundial.

- La vinculación con los clientes. Las redes proporcionan oportunidades para ampliar la conversación porque son inmediatas, pueden crear help-desk más personalizados, más eficientes y más ágiles.

- Las RSD favorece la identificación de riesgos que puedan tener un impacto negativo en la reputación de la empresa y además facilitan reaccionar de inmediato.

A pesar del auge de las RSD, todavía hay muchas empresas que tienen un dominio limitado en las redes sociales. En este sentido Uribe, Rialp y Llonch (2013) consideran que los creadores de políticas públicas y las agencias que promueven la creación de empresas y el desarrollo empresarial deberían desarrollar cursos y acciones que ayuden a las empresas a tener una mayor competencia en las redes sociales.

\subsection{El uso de las redes sociales para la difusión de contenidos}

Desde el contexto científico también se ha estudiado el uso de las RSD para la difusión de contenidos. Guallar et al. (2016) investigan el papel que tienen en el consumo de información de actualidad y concluyen que son vistas como un espa-

\begin{tabular}{|r|c|l|l|l|}
\hline & $\mathbf{2 0 1 6}$ & $\mathbf{2 0 1 7}$ & $\mathbf{2 0 1 8}$ & $\mathbf{2 0 1 9}$ \\
\hline YouTube & No disponible & $74 \%$ & $73 \%$ & $82 \%$ \\
\hline Facebook & $44 \%$ & $71 \%$ & $40 \%$ & $54 \%$ \\
\hline Instagram & $15 \%$ & $37 \%$ & $39 \%$ & Ya no existe \\
\hline Twitter & $24 \%$ & $44 \%$ & $32 \%$ & $31 \%$ \\
\hline Google & $21 \%$ & $35 \%$ & $27 \%$ & $28 \%$ \\
\hline Linkedln & $14 \%$ & $29 \%$ & $23 \%$ & \\
\hline
\end{tabular}

Tabla 3. Usuarios activos en España de las plataformas sociales globales durante los últimos cuatro años. Elaboración propia a partir de We Are Social (2016; 2017; 2018; 2019). 
cio adecuado para compartir noticias e informaciones sobre asuntos de interés público.

Por su parte, López-Pérez y Olvera-Lobo (2016) revisan el uso que los centros de investigación y las universidades públicas hacen de Facebook, Twitter y YouTube, para difundir sus investigaciones. Las autoras explican que, aunque se está empezando a utilizar Internet para "conversar" con los ciudadanos, ese diálogo no es fructífero ni en interacción, por la baja conectividad de los perfiles, ni en la difusión de resultados científicos, ya que representan una mínima parte de las publicaciones.

Asimismo, Zamarra (2015) analiza la presencia de los principales diarios españoles en las redes sociales y constata que las "redes sociales han trastocado radicalmente el panorama mediático" y han tenido que adaptarse a las redes sociales. Este cambio ha propiciado que los usuarios se hayan convertido en los propios "transmisores de la información en tiempo real" (p. 155).

\subsection{Uso de las redes sociales en otros ámbitos}

Sin embargo, existen otros indicios que demuestran la importancia que tienen las redes sociales para la opinión pública. Su utilización ha sido clave en campañas políticas, como el caso de Trump en EEUU (Rodríguez-Andrés, 2018) o el movimiento estudiantil chileno (Bacallao-Pino, 2016). También, ha permitido a los partidos políticos "conocer a sus simpatizantes, cuáles son sus principales problemas o preocupaciones, sus expectativas, la imagen que tienen del candidato" (Alonso, 2016, pp. 111).

En España los parlamentos autonómicos también se han incorporado progresivamente a las redes sociales digitales "como forma de potenciar una comunicación más directa con los ciudadanos" (Rodríguez-Andrés y Álvarez-Sabalegui, 2018, pp. 1001). Sin embargo "la mayoría de los perfiles siguen siendo usados de forma prioritaria como plataformas de difusión unidireccional de información" (pp. 1002).

Las redes sociales igualmente han sido cruciales para crear conciencia sobre problemas o causas y para difundir movimientos sociales como ocurrió en Twitter con "\#ShoutYourAbortion", que se creó para combatir el estigma que puede asociarse con el procedimiento médico del aborto (Ahmed, 2018).

Teniendo en cuenta el peso que tienen las RSD y las obligaciones de comunicación fijadas por CDTI, este artículo busca saber si se están utilizando como medio para dar a conocer los proyectos de I+D+i. La publicación a través de las RSD no es una obligación, pero ante la relevancia que tienen es fundamental conocer si se utilizan de forma complementaria.

\section{Objetivos}

La finalidad de la investigación es conocer qué RSD usan las empresas que reciben ayudas del CDTI y si las utilizan para dar a conocer las innovaciones que realizan. En concreto se busca: describir la presencia de las empresas en Facebook, Twitter, Instagram, YouTube, Pinterest, Google + y Linkedln; conocer: fecha de creación del perfil, número de seguidores, actividad de publicación, si utilizan las RSD para dar a conocer la financiación de CDTI y, en caso afirmativo, el contenido que se publica y si el mensaje es unitario en todas las redes; y estudiar las diferencias observadas entre unas redes y otras.

\section{Metodología}

La investigación está focalizada en las empresas que recibieron financiación de CDTI en enero de 2016 (CDTI, 2016). En este periodo se apoyan 70 proyectos, ejecutados por 65 compañías (anexo 1). Para el análisis, se ha tomado como referencia un periodo de veinticuatro meses (de enero de 2016 a diciembre de 2017), teniendo en cuenta que las ayudas de CDTI tienen una duración diferente: proyectos de I+D (de 12 a 36 meses); proyectos de innovación de CDTI (18 meses); proyectos NEOTEC (de 12 a 24 meses); proyectos de Innovación Global (hasta 24 meses); proyectos Estratégicos Cien (de 36 a 48 meses); proyectos Interempresas Internacional (de 12 a 36 meses). Así, durante el periodo examinado la empresa habrá logrado los primeros resultados significativos o habrá finalizado la iniciativa.

La localización de los perfiles de las empresas en las RSD se ha hecho observando si hay enlace directo en los sitios web y también se ha buscado directamente en las redes, utilizando

\begin{tabular}{|l|l|}
\hline \multicolumn{1}{|c|}{ Variable } & \multicolumn{1}{c|}{ Resultado } \\
\hline $\begin{array}{l}\text { La entidad tiene presencia } \\
\text { en la red social }\end{array}$ & $\mathrm{Si} / \mathrm{no}$ \\
\hline $\begin{array}{l}\text { Número de redes sociales } \\
\text { en las que tiene presencia }\end{array}$ & $\mathrm{No}$ \\
\hline La red social tiene contenido & $\mathrm{Si} / \mathrm{no}$ \\
\hline Fecha de creación & Año \\
\hline Seguidores & $\mathrm{No}$ \\
\hline Me gusta & $\mathrm{No}$ \\
\hline $\begin{array}{l}\text { Publicaciones durante } \\
\text { el periodo }\end{array}$ & $\mathrm{No}$ \\
\hline $\begin{array}{l}\text { Publicaciones que } \\
\text { abordan el proyecto }\end{array}$ & $\mathrm{Si} /$ no \\
\hline $\begin{array}{l}\text { Interactividad de las } \\
\text { publicaciones relacionadas } \\
\text { con el proyecto }\end{array}$ & No Me gusta \\
\hline
\end{tabular}

Tabla 4. Resumen de las variables revisadas. Elaboración propia. 
el nombre de la sociedad y/o la marca comercial.

En concreto, se han observado las nueve variables recogidas en la tabla 4.

Además, se ha utilizado el análisis de contenido, técnica que permite estudiar rigurosa y sistemáticamente la naturaleza de los mensajes (Krippendorff, 1990). Para identificar las publicaciones vinculadas con las ayudas se ha verificado la aparición de los términos: innovación, investigación y desarrollo e I+D, en español y en inglés (innovation, research and development, $R \& D)$, y se ha observado si estas palabras van acompañadas de una mención a CDTI o FEDER (origen de parte de los fondos).

\section{Resultados}

\subsection{Presencia en redes sociales}

Las empresas estudiadas tienen poca presencia en las RSD de referencia en España: Facebook, Twitter, Instagram, YouTube, Pinterest, Google + y Linkedln. Como se observa en la figura 1 , un 26,2\% no tiene perfil propio en ninguna RSD, un 20\% sólo tiene perfil propio en una y un 9,2\% está presente en dos. El 35,4\% de las compañías tiene perfil en más de tres plataformas y un $1,5 \%$ está en todas las RSD examinadas.

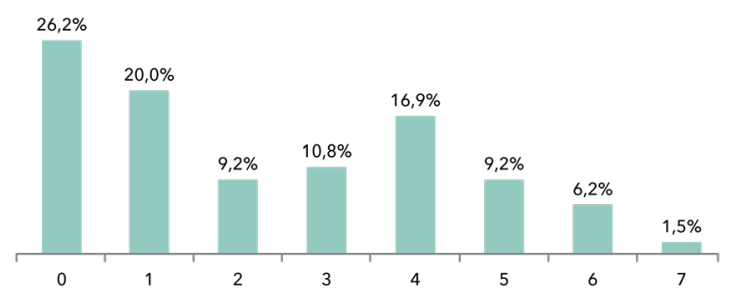

Figura 1. Presencia en las redes sociales. Elaboración propia.

\subsection{La comunicación a través de Facebook}

De las 65 empresas examinadas, se observa que el 43,1\% tiene perfil propio de Facebook, el 44,6\% no tiene perfil, el 10,8\% de las entidades no tiene perfil propio, pero tiene perfil del grupo, y el $1,5 \%$ tiene perfil con una sede de otro país.

El número de seguidores varía de unas empresas a otras (figura 2). El $57,2 \%$ de las entidades consideradas tienen menos de 500 seguidores, el 14,3\% tiene entre 501 y 1000 y el 28,6\% tienen más de 1.000 .

En términos absolutos, durante el periodo estudiado, predominan las empresas que han publicado más de 60 inserciones en total (50\%), seguido de las que han publicado entre 0 y 20

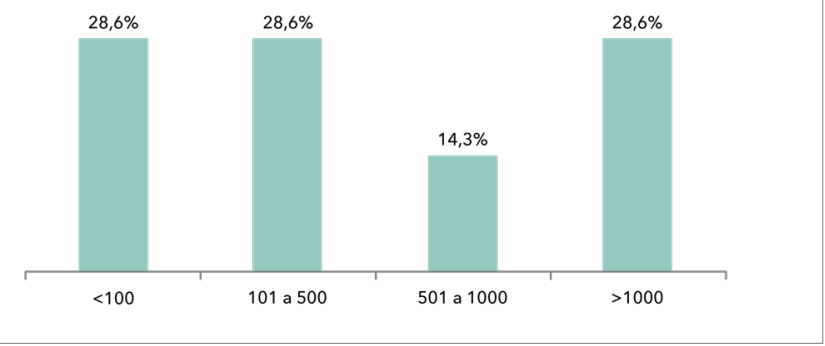

Figura 2. Número de seguidores en Facebook. Elaboración propia.

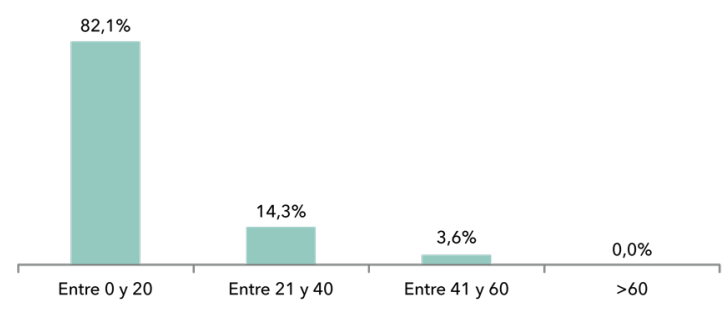

Figura 3. Media mensual de publicaciones durante el periodo observado en Facebook. Elaboración propia.

(35,7\%), entre 21 y 40 (7,1\%) o entre 41 y 60 (7,1\%).

Si tenemos en cuenta la media mensual, se aprecia que el $82,1 \%$ de las empresas en Facebook hacen menos de 20 publicaciones al mes y sólo el 3,6\% realizan entre 41 y 60 (figura 3).

En total se han revisado 5.891 inserciones y sólo la entidad Linkcare Health Services, S.L. ha realizado una publicación directamente relacionada con el proyecto (imagen 1), con un nivel de interactividad muy bajo (1 me gusta). La inserción no

\section{(1) Linkcare está aquí: ACCIÓ. \\ (C) 2 de junio de 2017 - (1) - (a)}

Presenting the experience of Linkcare with the CHINEKA cooperation program from CDTI Centro para el Desarrollo Tecnológico Industrial at the offices of ACCIÓ and part of the EUPIC's Sichuan mission to Barcelona

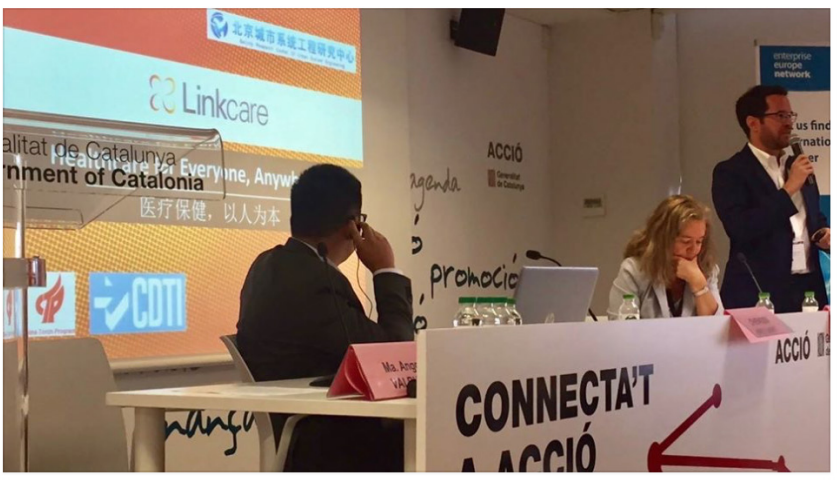

$$
\text { ¿3e gusta }
$$

$\square$ Comentar

$\Leftrightarrow$ Compartir

8 $>$

(1) 1

Imagen 1. Inserción en Facebook relacionada con el proyecto de Linkcare Health Services, S.L. Fuente: Perfil de Linkcare en Facebook. 
contiene el título del proyecto, pero nombra a CDTI, aparece su logo y enlaza con su perfil.

Aunque solo una entidad tiene una inserción relacionada directamente con el proyecto, un total de cinco empresas han publicado durante el periodo observado sobre otros proyectos CDTI (Blue Sostenible S.L; Thales Programas de Electrónica y Comunicaciones S.A; Calaf Tecniques Industrials S.L; Angulas Aguinaga S.A; y Compañía Española de Petróleos, S.A.U).

\subsection{La comunicación a través de Linkedln}

Esta red social es una de las plataformas más utilizadas. El $58,5 \%$ de las empresas tienen perfil en Linkedln, aunque solo el 52,6\% realiza "actualizaciones" con información. El número de seguidores es ligeramente superior al observado en Facebook y Twitter, ya que más del 44,8\% de las compañías supera los 500 seguidores.

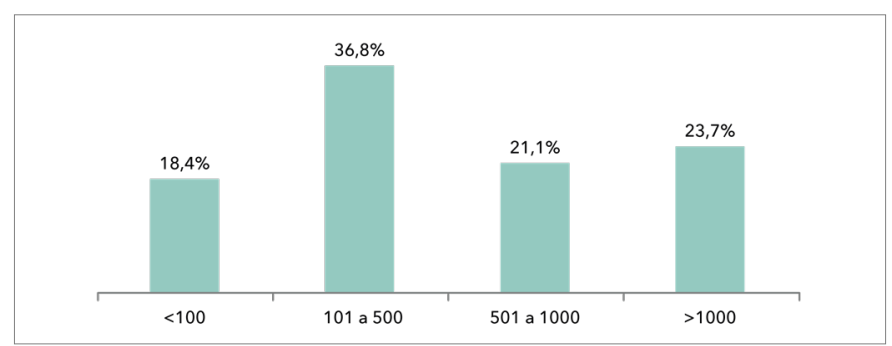

Figura 4. Número de seguidores en Linkedln. Elaboración propia.

Durante los 24 meses estudiados, el 55\% de la muestra que publica contenido tiene una actividad igual o inferior a 20 actualizaciones, el $20 \%$ ha realizado entre 21 y 40 y el $25 \%$ ha publicado más de 60 actualizaciones.

Solo la empresa Verdimed, S.A. inserta una publicación relacionada con el proyecto apoyado en julio de 2017 (imagen 2) y tiene 12 recomendaciones y un comentario. La inserción incluye el origen de los fondos, el título del proyecto y los logos de CDTI y Feder.

No obstante, hay entidades que publican información sobre sus procesos de innovación en general, o como Compañía Española de Petróleos, S.A.U., que no publica sobre el proyecto apoyado, pero realiza inserciones sobre otros proyectos de I+D (imagen 3).

\subsection{La comunicación a través de Twitter}

El 43,1\% de la muestra tiene perfil propio en Twitter, mientras que el $44,6 \%$ no lo tiene y el $12,3 \%$ de las entidades no tiene perfil propio, pero tiene uno del grupo empresarial al que pertenece (como el Grupo Siro, IVI Murcia o Vector Software Factory) o tiene el perfil de alguna sociedad vinculada (Grupo
Verdimed

verdimed 7 meses

Verdimed con la Innovación:

Verdimed con el apoyo del Centro para el Desarrollo Tecnológico Industrial (CDTI) y en colaboración con la Universidad Politécnica de Cartagena (UPCT) ha finalizado el primer año del proyecto RADUVCAGRO cuyo objetivo es el DESARROLLO DE UNA NUEVA TECNOLOGÍA SUSTITUTIVA A BIOCIDAS QUÍMICOS EN PRODUCTOS HORTOFRUTÍCOLAS (IDI-20160008), dicho proyecto se encuentra cofinanciado por el Fondo Europeo de Desarrollo Regional (FEDER) a través del programa operativo plurirregional de crecimiento inteligente.
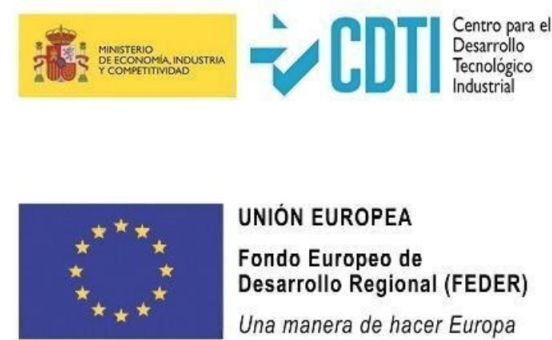

UNIÓN EUROPEA

Fondo Europeo de

Desarrollo Regional (FEDER)

Una manera de hacer Europa

12 recomendaciones $\cdot 1$ comentario

凹 $\Rightarrow$

Imagen 2. Publicación en Linkedln sobre el proyecto de la empresa Verdimed, S.A. Fuente: LinkedIn de la empresa.

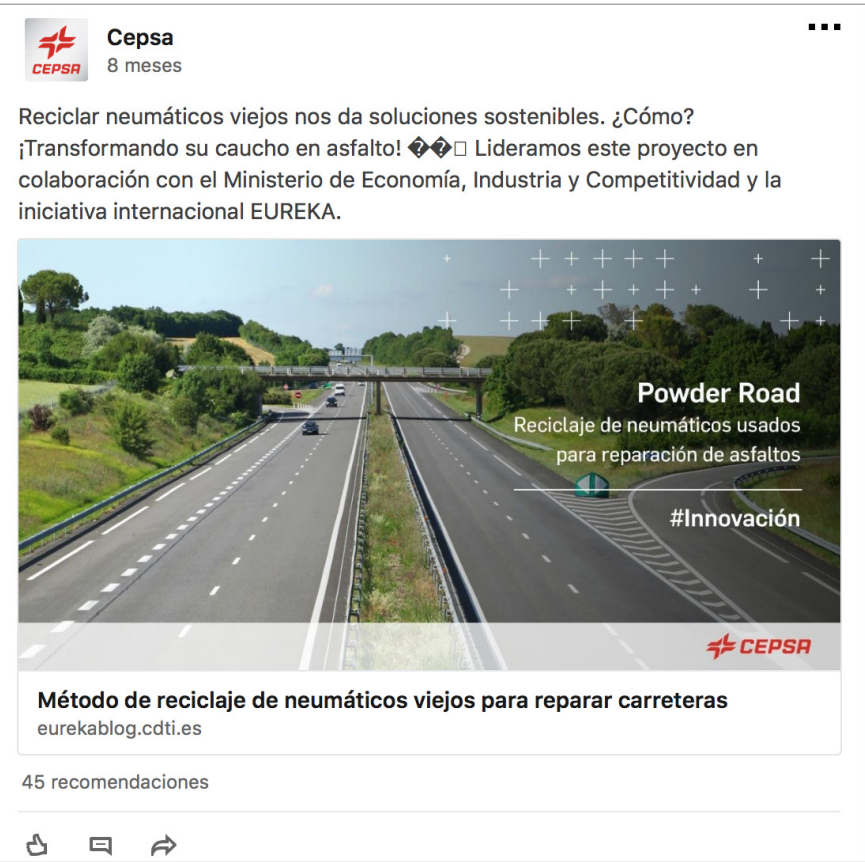

Imagen 3. Publicación en Linkedln de Cepsa sobre otros proyectos CDTI. Fuente: Linkedln de la empresa.

Rimar con Suavinex).

En el caso de Twitter podemos conocer la fecha de creación de los perfiles y se aprecia que tienen una edad media, dado que el 86,7\% se creó entre 2010 y 2015 (figura 5).

En lo referido al número de seguidores, se observa que en Twitter las empresas que tienen menos de 500 seguidores 


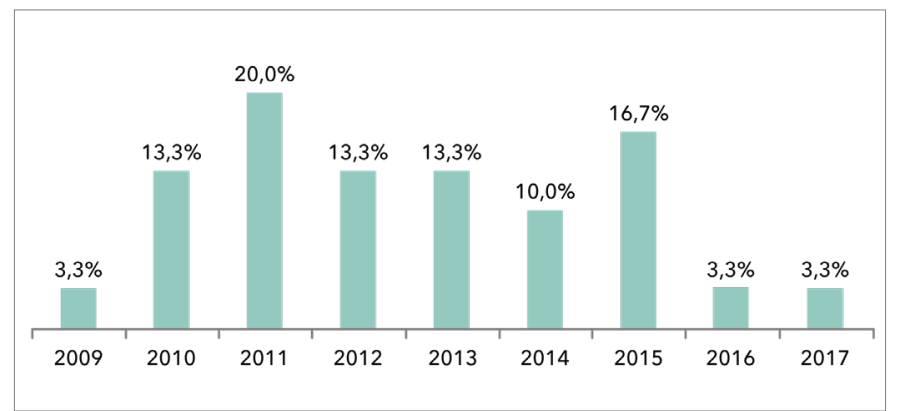

Figura 5. Fecha de creación de los perfiles en Twitter. Elaboración propia.

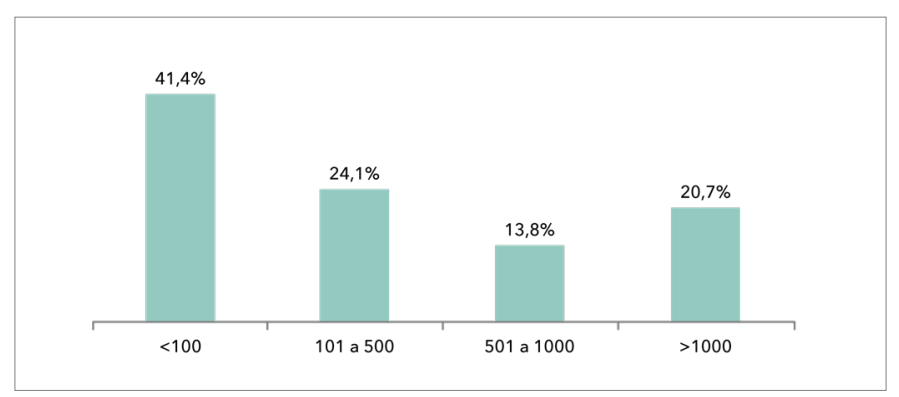

Figura 6. Número de seguidores en Twitter. Elaboración propia.

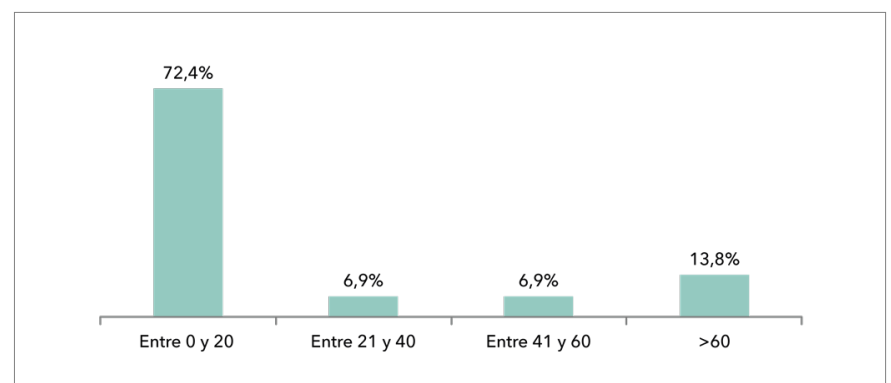

Figura 7. Media mensual de publicaciones en Twitter. Elaboración propia.

representan el 65,5\%, mientras que en Facebook alcanzaban el 57,2\% (figura 6).

En general, la frecuencia de publicación observada es media. El 64,3\% han realizado, durante los 24 meses estudiados, más de 60 inserciones, el 32,1\% han realizado entre 0 y 20 y el 3,6\% entre 41 y 60.

Si se estudia la media mensual, se aprecia que el 72,4\% de las empresas hacen menos de 20 publicaciones al mes y sólo el 13,8\% publica más de 60 tuits al mes (figura 7).

Entre los 14.680 tuits revisados no se ha encontrado ninguno relacionado con los proyectos. Además, la escasez de publicaciones impide la posibilidad de verificar si el mensaje en las dos redes sociales más populares, Facebook y Twitter, es unitario.

Si observamos el número de seguidores en las empresas que tienen presencia en las dos RSD, se aprecia que las compañías tienen más seguidores en Twitter que en Facebook (52,6\%). Sin embargo, en el caso de las entidades más activas, con presencia en las dos redes, el número de seguidores es muy superior en Facebook (Blue Sostenible S.L. 6.098 seguidores en Twitter y 16.221 en Facebook; Lacasa, S.A. 8.640 en Twitter y 77.318 en Facebook; y Compañía Española de Petróleos, S.A.U. 14.400 en Twitter y 63.800 en Facebook).

\subsection{La comunicación a través de YouTube}

YouTube es otra de las plataformas más populares, ya que la presencia de las empresas alcanza el $47,7 \%$ y el porcentaje de entidades que publican contenido supone el 93,5\%. Además, hay un $16,9 \%$ de la muestra que no tiene perfil propio en YouTube, pero que sí tiene perfil del grupo empresarial al que pertenece.

En YouTube también se puede conocer la fecha de incorporación a la plataforma (figura 8). No obstante, ese dato no está disponible en el $50 \%$ de los casos, aunque es posible tomar como referencia la fecha de publicación del primer vídeo. Teniendo en cuenta esa limitación, se observa que el 58,1\% de los perfiles se ha creado en los últimos tres años y sólo el

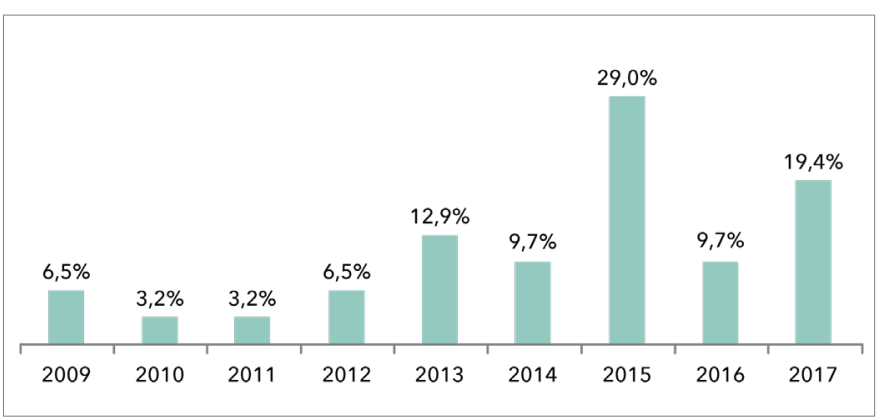

Figura 8. Fecha de incorporación a YouTube. Elaboración propia.

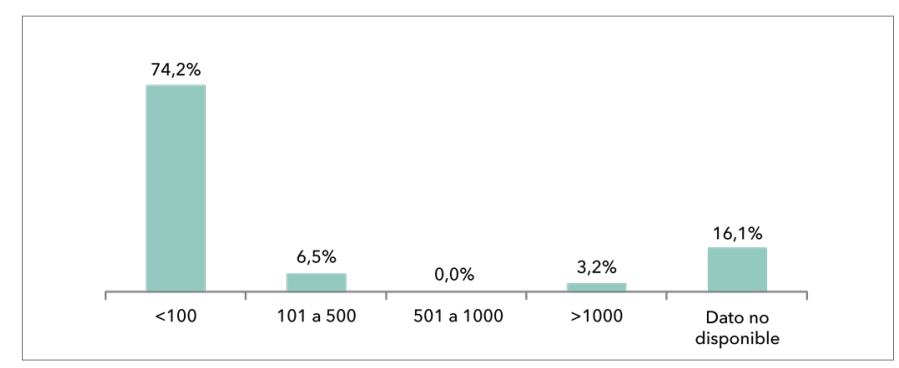

Figura 9. Número de seguidores en YouTube. Elaboración propia.

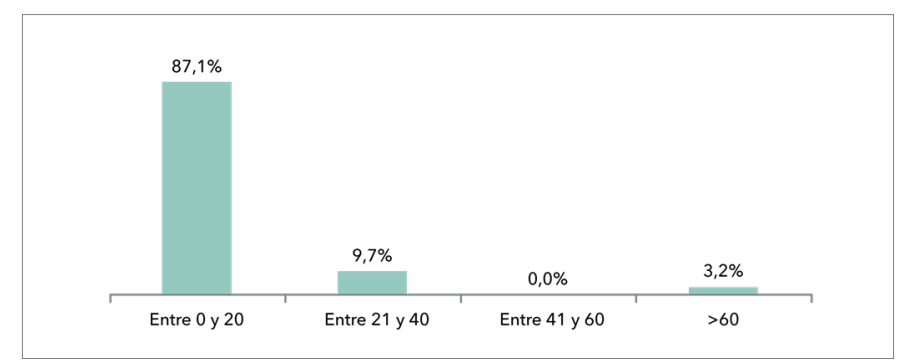

Figura 10. Vídeos publicados en YouTube. Elaboración propia. 


\section{9,4\% tiene más de cinco años.}

El número de seguidores es inferior al registrado en Twitter o Facebook. En YouTube el 74,2\% de las entidades tiene menos de 100 seguidores, el 6,5\% tienen entre 101 y 500 y el 3,2\% tiene más de 1000. Asimismo, este dato no está disponible en el 16,1\% de los casos (figura 9).

La frecuencia de publicación es baja (figura 10) en comparación con las RSD anteriores. Más del $87 \%$ de las empresas ha publicado, durante los veinticuatro meses estudiados, veinte o menos vídeos. Sólo el 3,2\% ha publicado más de 60 vídeos.

En esta red no hay ninguna inserción que esté relacionada con los proyectos apoyados, sin embargo, hay entidades, como SPB o Cepsa, que publican información sobre sus innovaciones en general.

\subsection{La comunicación a través de Instagram}

El 9,3\% de las empresas examinadas tiene perfil en Instagram, mientras que el 81,5\% no tiene presencia, o la tiene a través de un perfil del grupo (7,7\%) o de otro país (1,5\%). Asimismo, no todas las empresas con perfil tienen contenido, pues el 16,7\% no ha realizado ninguna publicación.

Por otra parte, ninguna de las empresas supera los 500 seguidores en esta plataforma. El 50\% tiene menos de 100 seguidores y el otro 50\% posee entre 101 y 500, teniendo la empresa con más seguidores 362.

Al profundizar en la actividad, se aprecia que es muy baja. Sólo cuatro empresas tienen alguna publicación entre enero de 2016 y diciembre de 2017 y ninguna entidad ha realizado más de 100 inserciones durante esos 24 meses. Tres empresas han publicado entre 21 y 40 publicaciones y solo una tiene más de 60. En ningún caso se ha utilizado esta red para comunicar la ayuda recibida de CDTI.

\subsection{La comunicación a través de Pinterest}

El porcentaje de entidades que comunican a través de Pinterest es muy bajo. Sólo 5 empresas (el 7,7\%) tienen perfil propio, una entidad centraliza la comunicación a través de la matriz (Siro El Espinar) y otra lo tiene centralizado a través de otra sociedad (Lacasa).

En esta plataforma el número de seguidores es bajo. El 80\% de las empresas tienen menos de 100 seguidores y sólo una empresa, Aqua E-Solutions, S.A., supera los 600.

Teniendo en cuenta el número de pines totales de cada empresa, se observa que hay una gran diferencia. Aqua E-Solutions, con más de 3.000 pines, y Angulas Aguinaga, con 979, son las entidades con mayor producción. El resto ha publicado menos de 350 pines

En total se han examinado 4.737 pines y ninguna publicación está relacionada con el proyecto apoyado por CDTI.

\subsection{La comunicación a través de Google +}

De las entidades estudiadas, el 21,5\% tiene perfil en Google + (se incluye una empresa que tiene una comunidad privada), el $66,2 \%$ no tiene, el 6,2\% tiene la comunicación centralizada a través del perfil de grupo y el otro 6,2\% tiene perfil de otros países. Sin embargo, un $28,6 \%$ de las empresas con perfil no tiene ningún contenido publicado (Farmalider, Industrias Metalúrgicas Jem y Singular Meaning) o no es accesible (la comunidad privada de (epsa).

A excepción de Cepsa y Bluemove Carsharing, con 297 y 126 seguidores, el resto de entidades tiene menos de 15 seguidores. Cabe destacar que de Aqua E-Solutions e Hidro Rubber Ibérica no hay datos disponibles del número de seguidores.

La actividad de publicación en esta plataforma es muy baja. El 90\% de las empresas ha publicado menos de diez inserciones. La única empresa que registra una producción superior es Aqua E-Solutions con 44 publicaciones.

Ninguna entidad ha utilizado esta red social para comunicar el apoyo recibido por CDTI.

\subsection{Comparación entre redes sociales}

Teniendo en cuenta los datos recogidos (tabla 5), se observa que Linkedln es la RSD en la que hay más perfiles de las empresas, seguida de YouTube, Facebook, Twitter, Google +, Instagram y Pinterest. No obstante, cerca del $47 \%$ no los utilizan para publicar noticias.

Desde la vertiente de los seguidores, Linkedln (44,7\%) y Facebook (42,9\%) son las RSD con más empresas que acumulan más de 500 seguidores, mientras que Pinterest y Google + son las que tienen menos seguidores.

Atendiendo al número de publicaciones, hay una gran brecha entre Twitter y Facebook respecto a otras redes como Google + o YouTube, en las que se realizan muy pocas inserciones.

Para finalizar, las empresas solo han utilizado las plataformas de Facebook y Linkedln para realizar alguna comunicación relacionada con los proyectos apoyados y, al tratarse de entidades diferentes, es imposible verificar si el mensaje que transmiten las empresas en las diferentes redes sociales es unitario.

\section{Discusión y conclusiones}

Los estudios previos han mostrado que las redes sociales digitales son un espacio útil para entablar un diálogo fructífero 


\begin{tabular}{|c|c|c|c|c|c|c|c|}
\hline & Facebook & Twitter & Instagram & YouTube & Pinterest & Google + & Linkedln \\
\hline *Perfil propio & $43,1 \%$ & $43,1 \%$ & $9,2 \%$ & $47,7 \%$ & $7,7 \%$ & $21,5 \%$ & $58 \%$ \\
\hline *Perfil con contenido & $89,3 \%$ & $89,3 \%$ & $83,3 \%$ & $93,5 \%$ & $100 \%$ & $71,4 \%$ & $52,6 \%$ \\
\hline \multicolumn{8}{|c|}{ *Seguidores } \\
\hline$<100$ & $28,6 \%$ & $41,4 \%$ & $50 \%$ & $74,2 \%$ & $80 \%$ & $71,4 \%$ & $18,4 \%$ \\
\hline 101 a 500 & $28,6 \%$ & $24,1 \%$ & $50 \%$ & $6,5 \%$ & & $14,3 \%$ & $36,8 \%$ \\
\hline 501 a 1000 & $14,3 \%$ & $13,8 \%$ & & & $20 \%$ & & $21,1 \%$ \\
\hline$>1000$ & $28,6 \%$ & $20,7 \%$ & & $3,2 \%$ & & & $23,7 \%$ \\
\hline Dato no disponible & & & & $16,1 \%$ & & $14,3 \%$ & \\
\hline \multicolumn{8}{|c|}{ *Publicaciones totales en 24 meses } \\
\hline Entre 0 y 20 & $35,7 \%$ & $32,1 \%$ & $20 \%$ & $87,1 \%$ & $20 \%$ & $90 \%$ & $55 \%$ \\
\hline$>20 y<40$ & $7,1 \%$ & & $60 \%$ & $9,7 \%$ & & & $20 \%$ \\
\hline$>40 y<60$ & $7,1 \%$ & $3,6 \%$ & & & & $10 \%$ & \\
\hline$>60$ & $50,0 \%$ & $64,3 \%$ & $20 \%$ & $3,2 \%$ & $80 \%$ & & $25 \%$ \\
\hline $\begin{array}{l}\text { *Inserciones } \\
\text { relacionadas }\end{array}$ & 1 & 0 & 0 & 0 & 0 & 0 & 1 \\
\hline
\end{tabular}

Tabla 5. Resumen de las principales variables estudiadas por red social. Elaboración propia

con la población y fomentar su participación en las cuestiones de interés público (Rodríguez-Andrés y Álvarez-Sabalegui, 2018). Asimismo, también se ha constatado que las redes sociales tienen una gran influencia en la propagación de movimientos sociales (Bacallao-Pino, 2016; Ahmed, 2018). Tal es su importancia que, tanto las empresas como las administraciones públicas, las están empleando para mejorar su diálogo con los públicos y las están incorporando de forma progresiva dentro de sus planes de comunicación.

Sin embargo, los datos aportados en la presente investigación muestran que la presencia de las empresas innovadoras en las redes sociales es modesta, coincidiendo con las aportaciones de Sixto, Aguado y Riveiro (2017), a pesar de que se trata de un espacio adecuado para compartir noticias de interés público (Guallar et al, 2016).

Las empresas prácticamente no utilizan las RSD para comunicar los proyectos de investigación apoyados por CDTI. Por ello, se puede concluir que el diálogo entre las empresas innovadoras y el público en general continúa siendo muy bajo, al igual que ocurre con la difusión de la investigación en las universidades (López y Olvera, 2016).

El nivel de penetración de las redes sociales en España es superior a la media mundial y está registrando crecimientos muy significativos (We Are Social, 2019). Por ello, su inclusión dentro de los requisitos de comunicación determinados por CDTI podría derivar en un mayor impacto de la difusión. Asimismo, la concreción de los contenidos a publicar y de las plataformas a utilizar repercutiría positivamente en la efecti- vidad de la comunicación.

En la misma dirección, el apoyo de los organismos públicos en la definición e implementación de la política de comunicación a través de las redes sociales se ha considerado útil para lograr que las empresas tengan una mayor competencia en las RSD (Uribe, Rialp y Llonch, 2013). En este sentido, sería de interés que los organismos gestores de las ayudas apoyaran a las empresas innovadores en la definición de su plan de comunicación online.

En España, YouTube, Facebook e Instagram son las redes sociales con más usuarios (We Are Social, 2019). Sin embargo, no hay estudios que analicen qué redes sociales son más adecuadas para la difusión de la innovación. Por ello, futuras líneas de investigación podrían consistir en estudiar qué redes sociales son más apropiadas para dar a conocer los resultados de las investigaciones apoyadas con fondos públicos.

\section{Referencias}

Ahmed, W. (2018). Public health implications of\# ShoutYourAbortion. Public health, 163, 35-41.

Alonso González, M. (2016). Opinión pública y web 2.0. Las redes digitalizan el barómetro político en España. Revista mexicana de opinión pública, (21), 95-113.

Andreu Pinillos, A. (2015). Transformar la cultura corporativa de las empresas. Revista Telos, 101, 9-11.

Bacallao-Pino, L. M. (2016). Redes sociales, acción colectiva y elecciones: los usos de Facebook por el movimiento estudiantil chileno durante la campaña electoral de 2013. Palabra Clave, 19(3), 810-837. 
Beltrán-Orenes, P., y Martínez-Pastor, E. (2016). Grado de cumplimiento de las leyes de transparencia, acceso y buen gobierno y de reutilización de los datos de contratación de la administración central española. El Profesional de la Información, 25(4), 557-567.

Centro para el Desarrollo Industrial Tecnológico (2015). Proyectos aprobados. Perspectiva CDTI: revista de innovación tecnológica, (46), 4p.

Comunicación de la Comisión 2014/C 198/01.Marco sobre ayudas estatales de investigación y desarrollo e innovación. Diario Oficial de la Unión Europea, 27 de junio de 2014.

Cruz-Rubio, C. N. (2017). Ley de transparencia y grandes empresas en España. Madrid: Transparency International España. ISBN: 9788469743089.

Ley 38/2003, de 17 de noviembre, General de Subvenciones. BOE, n. 276, 18 de noviembre de 2003 .

Ley $19 / 2013$, de 9 de diciembre, de transparencia, acceso a la información pública y buen gobierno. BOE, n. 295, 10 de diciembre de 2013.

Fundación Telefónica (2014). Desarrollo empresarial y redes sociales. El caso de las microempresas españolas. Madrid: Fundación Telefónica.

Guallar, J., Suau, J., Ruiz-Caballero, C., Sáez, A., y Masip, P. (2016). Re-dissemination of news and public debate on social networks. El profesional de la información, 25(3), 358-366.

Interactive Advertising Bureau IAB (2017). Estudio Anual de Redes Sociales.

Krippendorff, K. (1990). Metodología de análisis de contenidos. Teoría y práctica. Barcelona. Editorial Paidós.

López-Pérez, L., y Olvera-Lobo, M. D. (2016). Comunicación pública de la ciencia a través de la web 2.0. El caso de los centros de investigación y universidades públicas de España. El profesional de la información, 25(3), 441-448.

Real Decreto 887/2006, de 21 de julio, por el que se aprueba el Reglamento de la Ley 38/2003, de 17 de noviembre, General de Subvenciones. BOE, n. 176, 25 de julio de 2006.

Reglamento № 1303/2013 del Parlamento Europeo y del Consejo de 17 de diciembre de 2013 por el que se establecen disposiciones comunes relativas al Fondo Europeo de Desarrollo Regional, al Fondo Social Europeo, al Fondo de Cohesión, al Fondo Europeo Agrícola de Desarrollo Rural y al Fondo Europeo Marítimo y de la Pesca, y por el que se establecen disposiciones generales relativas al Fondo Europeo de Desarrollo Regional, al Fondo Social Europeo, al Fondo de Cohesión y al Fondo Europeo Marítimo y de la Pesca, y se deroga el Reglamento (CE) no 1083/2006 del Consejo. BOE, n. 347, 20 de diciembre de 2013.

Reglamento n 821/2014 de la Comisión de 28 de julio de 2014 por el que se establecen disposiciones de aplicación del Reglamento (UE) no 1303/2013 del Parlamento Europeo y del Consejo en lo que se refiere a las modalidades concretas de transferencia y gestión de las contribuciones del programa, la presentación de información sobre los instrumentos financieros, las características técnicas de las medidas de información y comunicación de las operaciones, y el sistema para el registro y el almacenamiento de datos. Diario Oficial de la Unión Europea, 29 de julio de 2014.

Rodríguez-Andrés, R. (2018). Trump 2016: ¿presidente gracias a las redes sociales? Palabra Clave, 21(3), 831-859.

Rodríguez-Andrés, R., y Álvarez-Sabalegui, D. (2018). Presencia y acti- vidad de los parlamentos autonómicos en las redes sociales: pocos avances en el fomento de la participación ciudadana. El profesional de la información, 27(5), 994-1003.

Ruiz Iniesta, C. (2012). La comunicación empresarial en redes sociales. El caso de las cinco mayores empresas españolas. Razón y palabra, n 79.

Sixto García, J., Aguado Domínguez, N., y Riveiro Castro, R. (2017). Presencia 2.0 de las pymes gallegas: niveles de participación y engagement con los usuarios. Revista Latina de Comunicación Social, 72 , 47-68.

Uribe Saavedra, F., Rialp Criado, J., y Llonch Andreu, J. (2013). El uso de las redes sociales digitales como herramienta de marketing en el desempeño empresarial. Cuadernos de administración, 26(47).

Vilaplana-Aparicio, M. J., Martín-Llaguno, M., y Iglesias-García, M. (2018). How should companies that receive R\&D\&l funds communicate? Legal requirements in Spain. El profesional de la información, 27 (3), 633-640.

We are Social (2016) Global Digital Report 2016. Recuperado de https://wearesocial.com/special-reports/digital-in-2016

We are Social (2017) Global Digital Report 2017. Recuperado de https://wearesocial.com/special-reports/digital-in-2017-globaloverview

We are Social (2018) Global Digital Report 2018. Recuperado de https://wearesocial.com/blog/2018/01/global-digital-report-2018

We are Social (2019) Global Digital Report 2019. Recuperado de https://wearesocial.com/global-digital-report-2019

Zamarra López, M. M. (2015). Análisis de los diarios digitales españoles más influyentes y su presencia en las redes sociales/Analysis of the most influential Spanish digital newspapers and their presence on social networks. Estudios sobre el Mensaje Periodístico, 21, 155-170.

\section{CV}

Maria J. Vilaplana-Aparicio. Doctora por la Universidad de Alicante. Licenciada en Publicidad y Relaciones Públicas. Profesora del Departamento de Comunicación y Psicología Social de la Universidad de Alicante. Imparte docencia en el Grado de publicidad y relaciones públicas. Compagina la docencia con la consultoría en innovación y realiza cursos y talleres relacionados con la I+D+i. https://cvnet.cpd.ua.es/curriculum-breve/ es/vilaplana-aparicio-maria-jose/29135

Mar Iglesias-García. Periodista y profesora del Departamento de Comunicación y Psicología Social de la Universidad de Alicante. Imparte docencia en el grado de publicidad y relaciones públicas y en el grado de turismo. Colabora en el Proyecto de Investigación Creación y contenido interactivo en la comunicación de información audiovisual: audiencias, diseño, sistemas y formatos, financiado por el Ministerio de Economía y Competitividad. Es vicepresidenta de la Corporació Valenciana de Mitjans de Comunicació (CVMC) y directora de RàdioUA. https://cvnet.cpd.ua.es/curriculum-breve/es/iglesias-gar- 
cia-mar/1783

Marta Martín-Llaguno. Catedrática de Comunicación. Ha publicado medio centenar de artículos en revistas indexadas y ha participado en más de veinte proyectos competitivos (europeos, nacionales y autonómicos). Ha sido profesora visitante en la Universidad de Piura (Perú), en la Universidad Católica de Buenos Aires e investigadora visitante del IESE Business School y de la City University of New York (USA). Es miembro del Jurado de Autocontrol de la Publicidad. Actualmente se encuentra en comisión de servicios especiales. https://cvnet. cpd.ua.es/curriculum-breve/es/martin-llaguno-marta/857

\section{Anexo: Empresas estudiadas}

\begin{tabular}{|c|c|}
\hline & Empresa \\
\hline 1 & Angulas Aguinaga S.A \\
\hline 2 & Aperitivos Matarile S.A. \\
\hline 3 & Exafan, S.A. \\
\hline 4 & Farmalider, S.A. \\
\hline 5 & Iniciativas Alimentarias S.A. \\
\hline 6 & IVI Murcia S.L. \\
\hline 7 & Laboratorio Arago S.L. \\
\hline 8 & Lacasa, S.A. \\
\hline 9 & Linkcare Health Services S.L. \\
\hline 10 & $\begin{array}{l}\text { S.Coop. Agrícola Aragonesa del Ebro conservación y } \\
\text { vida útil de los piensos. }\end{array}$ \\
\hline 11 & Sat Bernaus Petit Num 1130 Cat \\
\hline 12 & Siro El Espinar S.L. \\
\hline 13 & Vall Companys, S.A. \\
\hline 14 & Verdimed, S. A. \\
\hline 15 & Fabricante de Contenedores Fadeco, S.A. \\
\hline 16 & Filtros Industriales S.L. \\
\hline 17 & Fomento de Construcciones y Contratas, S.A. \\
\hline 18 & Geotecnia y Cimientos, S.A. \\
\hline 19 & Industrias Metalúrgicas Jem S.A. \\
\hline 20 & Ingeniería y Equipamientos S.A. \\
\hline 21 & Sacyr Construcción S.A. \\
\hline 22 & Talleres Mecacontrol,S.L. \\
\hline 23 & Vak Kimsa, S.A. \\
\hline 24 & Aqua E-Solutions, S.A. \\
\hline 25 & Blue Sostenible S.L. \\
\hline 26 & Cirprotec, S.L. \\
\hline 27 & Entelgy Consulting S.A. \\
\hline 28 & Gamesa Electric Power Systems S.L. \\
\hline 29 & Hotwords España S.L. \\
\hline 30 & Lazarus Technology S.L. \\
\hline 31 & Luz Wavelabs S.L. \\
\hline 32 & Mier Comunicaciones, S.A. \\
\hline
\end{tabular}

\begin{tabular}{|c|c|}
\hline 33 & Professional Answer S.L. \\
\hline 34 & Robot, S.A. \\
\hline 35 & SHS Consultores, S.L. \\
\hline 36 & Singular Meaning S.L. \\
\hline 37 & $\begin{array}{l}\text { Thales Programas de Electrónica y Comunicaciones } \\
\text { S.A. }\end{array}$ \\
\hline 38 & Ulma Manutención, S.Coop. \\
\hline 39 & Unifit Online S.L. \\
\hline 40 & Vector Software Factory S.L. \\
\hline 41 & Al Farben, S.A. \\
\hline 42 & Andaluza Tratamientos Higiene S.A. \\
\hline 43 & Calaf Tecniques Industrials S.L. \\
\hline 44 & Castey Global Sociedad Limitada \\
\hline 45 & Cobert Tejas Ibérica S.L. \\
\hline 46 & Compañía Española de Petróleos, S.A.U. \\
\hline 47 & Euroatomizado, S.A. \\
\hline 48 & Exclusivas Rimar, S.L. \\
\hline 49 & Fundiciones Mecacontrol S.L. \\
\hline 50 & Gocam Empresas Plásticas S.L. \\
\hline 51 & Hidro Rubber Iberica Sociedad Anónima \\
\hline 52 & Ingelia S.L. \\
\hline 53 & IQAP Masterbatch Group S.L \\
\hline 54 & Panel Fijaciones Sociedad Cooperativa \\
\hline 55 & Pavimentos Asfálticos Lario S.L. \\
\hline 56 & Plásticos Hidrosolubles, S.L. \\
\hline 57 & Practicel S.L. \\
\hline 58 & $\begin{array}{l}\text { Productos Plásticos Performantes 3P Sociedad Anó- } \\
\text { nima }\end{array}$ \\
\hline 59 & Retineo Ingeniería S.L. \\
\hline 60 & Soler \& Palau Research S.L. \\
\hline 61 & Suavizantes y Plasti cantes Bituminosos, S.L. \\
\hline 62 & Tecnoquim del Vallés S.L. \\
\hline 63 & Torrecid S.A. \\
\hline 64 & Urbiliza Renovables S.L. \\
\hline 65 & Ursa Insulation S.A. \\
\hline
\end{tabular}

